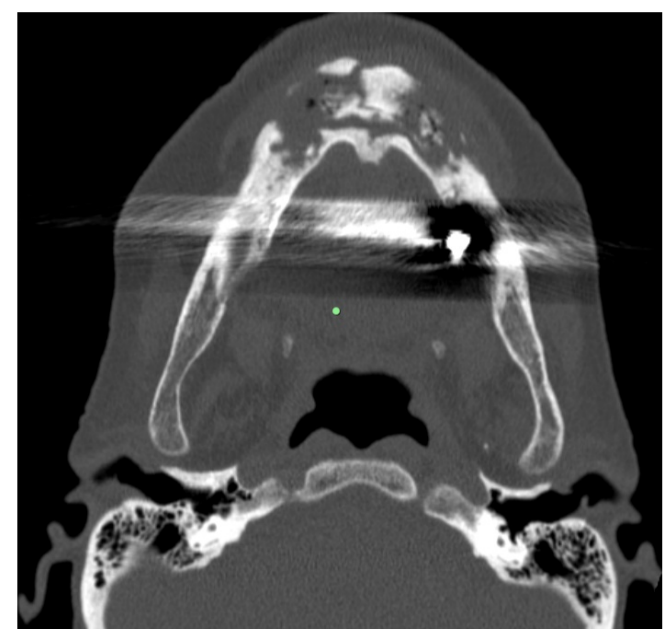

\title{
OSTEONECROSIS OF THE JAW (ONJ) IN BREAST CANCER PATIENTS: EFFECT OF PREVENTIVE MEASURES IN A MONOINSTITUTIONAL EXPERIENCE
}

Paola Brigo, Vittorio Fusco ${ }^{1}$, Antonella Fasciolo ${ }^{1}$, Maura Rossi ${ }^{1}$, Pamela Francesca Guglielmini ${ }^{1}$, Daniela Tartara ${ }^{1}$, Pier Luigi Piovano ${ }^{1}$, Elisabetta Ferrero ${ }^{1}$, maura vincenti ${ }^{1}$, Carmela Riccio ${ }^{1}$, Iolanda De Martino, Manuela Alessio ${ }^{1}$

1 Azienda Ospedaliera SS. Antonio e Biagio e Cesare Arrigo

Funding: The author(s) received no specific funding for this work.

Potential competing interests: The author(s) declared that no potential competing interests exist.

\section{Abstract}

Charts of all breast cancer patients affected by bone metastases observed at the Oncology Unit of Alessandria Hospital in years 2005-2020 have been reviewed. Osteonecrosis of the Jaw (ONJ) frequency after antiresorptive treatment (bisphosphonates and/or denosumab) was investigated in breast cancer patients undergoing preventive (before start of treatment) and/or screening (during treatment) evaluations, including oral inspection and panoramic dental radiography. Patients received antiresorptive agents according to international guidelines, that partially recommended shorter and tailored duration of antiresorptive treatment (instead of indefinite prolonged administration, till to deterioration of Performance Status - as advised by older guidelines) only after 2008. We examined charts of 280 patients with bone metastases receiving bisphosphonates (mostly zoledronic acid or pamidronate) or denosumab or sequences of drugs, and evaluated for oral health, of which 196 treated after 2006, and undergoing pre-treatment evaluation. Out of 280 total patients, 24 cases of Osteonecrosis of the Jaw (ONJ) were registered among Alessandria Hospital breast cancer patients. However, 9 cases were detected among 84 cases starting antiresorptive treatment without preventive visit, and 15 cases were found among 169 cases receiving dental visit before treatment beginning 
(15/169, 8.8\%). Our preliminary data do not show the decrease of the risk of ONJ in breast cancer patients treated in the latest decade, that we expected due to generally less intensive schedules of antiresorptive drugs and due to adoption of measure of pre-treatment visit. Further evaluations have been planned to investigate if higher ONJ risk was associated to single drug (denosumab versus zoledronic acid versus pamidronate) or sequences; prolonged treatment (> 2 years); pre-existing oral disease (periodontal disease, need for tooth extraction, ill fitting dentures); and other potential predictive factors

Background: Breast cancer incidence is about 120 cases on 100.000/year. Bone is the most frequent site of metastases from breast and the presence of Skeletal Related Events (SRE) increases morbidity. The treatment is based on chemotherapy, endocrine therapy, radiotherapy and recently also target therapy agents (trastuzumab, lapatinib, pertuzumab, bevacizumab, everolimus, cycline inhibitors, and so on). These therapies are frequently associated with antiresorptive agents, such as Bisphosphonates (BPs), including pamidronate, zoledronic acid, ibandronate, or an antiRANKL agent, denosumab (at monthly doses of $120 \mathrm{mg}$ ). All these agents can reduce the risk of Skeletal Related Events (SREs) and to delay SRE onset. However, they are associated with occurrence of Osteonecrosis of Jaw (ONJ) in 1-18\% of breast cancer patients ${ }^{[1]}$. Preventive (risk reduction) measures before BP and denosumab treatment (dental visit, dental RX, eventual teeth extractions, dental and denture care) have been largely recommended since $2007^{[2]}$ and largely applied in later years ${ }^{[3][4]}$. Recommendations for shorter treatment (1-2 years and then tailoring) were published since $2008^{[5]}$ but other guidelines went on proposing continuous long-term treatment ${ }^{[6]}$.

Materials and Methods: We reviewed all breast cancer patients affected by bone metastases observed by our Hospital in years 2005-2020. They were observed by a multidisciplinary team (including maxillofacial surgeons, dentists, hematologists, oncologists, nurses, radiologists, nuclear medicine and infective disease specialists) before start of antiresorptive treatment (if possible) and during that treatment (in all the cases). After 2006 patients underwent a baseline oral assessment (dental visit, dental panoramic Rx, eventual tooth avulsion or dental care) before start of treatment. Patients were consequently classified in 3 groups: a)"historical group" (patients starting BP treatment before 2005) b)"screening group" (patients starting therapy without preventive measures, since 2005) c) "prevention group" (patients who started antiresorptive drugs after preventive visit). The latter group mostly underwent shorter treatment, according to latest treatment guidelines (2 years of antiresorptive treatment and then tailoring on the base of single patient assessment).

Results: We followed 280 breast cancer patients treated with BPs and/or denosumab. ONJ was observed in 24/280, cases (8.5\%). The ONJ frequency in the several subgroups was: in 6/60 patients (10.0\%) in group a, in 3/24 patients (12.5 $\%)$ in group $b$, and in $15 / 169$ patients from group $c(8.8 \%)$.

Furthermore, 15 other $\mathrm{ONJ}$ cases in breast cancer patients were referred to the multidisciplinary group by neighboring hospitals (not evaluated by the group before start of treatment and not included in this analysis) after treatment with BPS and/or denosumab.

Conclusions: Preliminary evaluations seem to show a reduction of the ONJ rate smaller than expected in breast cancer patients treated with BPs and/or denosumab in latest decade, even if preventive measures are applied and shorter duration treatments were generally (non totally) adopted. Our evaluation needs further investigations with eventual 
multivariate analyses, to verify impact of type of drugs (denosumab versus zoledronic acid versus pamidronate versus sequence of drugs), prolonged treatment (> 2 years), pre-existing oral disease (periodontal disease, need for tooth extraction, ill fitting dentures), and other potential predictive factors.

\section{References}

1. 'Terry L. Ng, Megan M. Tu, Mohammed F. K. Ibrahim, Bassam Basulaiman, et al. (2020). Long-term impact of bonemodifying agents for the treatment of bone metastases: a systematic review. Support Care Cancer, vol. 29 (2), 925-943. doi:10.1007/s00520-020-05556-0.

2. ^(2007). American Association of Oral and Maxillofacial Surgeons Position Paper on Bisphosphonate-Related Osteonecrosis of the Jaws. Journal of Oral and Maxillofacial Surgery, vol. 65 (3), 369-376. doi:10.1016/j.joms.2006.11.003.

3. 'C.I. Ripamonti, M. Maniezzo, T. Campa, E. Fagnoni, et al. (2009). Decreased occurrence of osteonecrosis of the jaw after implementation of dental preventive measures in solid tumour patients with bone metastases treated with bisphosphonates. The experience of the National Cancer Institute of Milan. Annals of Oncology, vol. 20 (1), 137-145. doi:10.1093/annonc/mdn526.

4. ^Alberto Bedogni, Giuseppina Campisi, Alessandro Agrillo, Vittorio Fusco. (2013). Raccomandazioni clinicoterapeutiche sull'osteonecrosi delle ossa mascellari associata a bisfosfonati e sua prevenzione. Cleup.

5. 'M. Aapro, P.A. Abrahamsson, J.J. Body, R.E. Coleman, et al. (2008). Guidance on the use of bisphosphonates in solid tumours: recommendations of an international expert panel. Annals of Oncology, vol. 19 (3), 420-432. doi:10.1093/annonc/mdm442.

6. 'Catherine Van Poznak, Mark R. Somerfield, William E. Barlow, J. Sybil Biermann, et al. (2017). Role of BoneModifying Agents in Metastatic Breast Cancer: An American Society of Clinical Oncology-Cancer Care Ontario Focused Guideline Update. JCO, vol. 35 (35), 3978-3986. doi:10.1200/jco.2017.75.4614. 